\title{
Desarrollo del pensamiento computacional basado en diseño de tecnología educativa
}

Development of computational thinking based on the design of educational technology

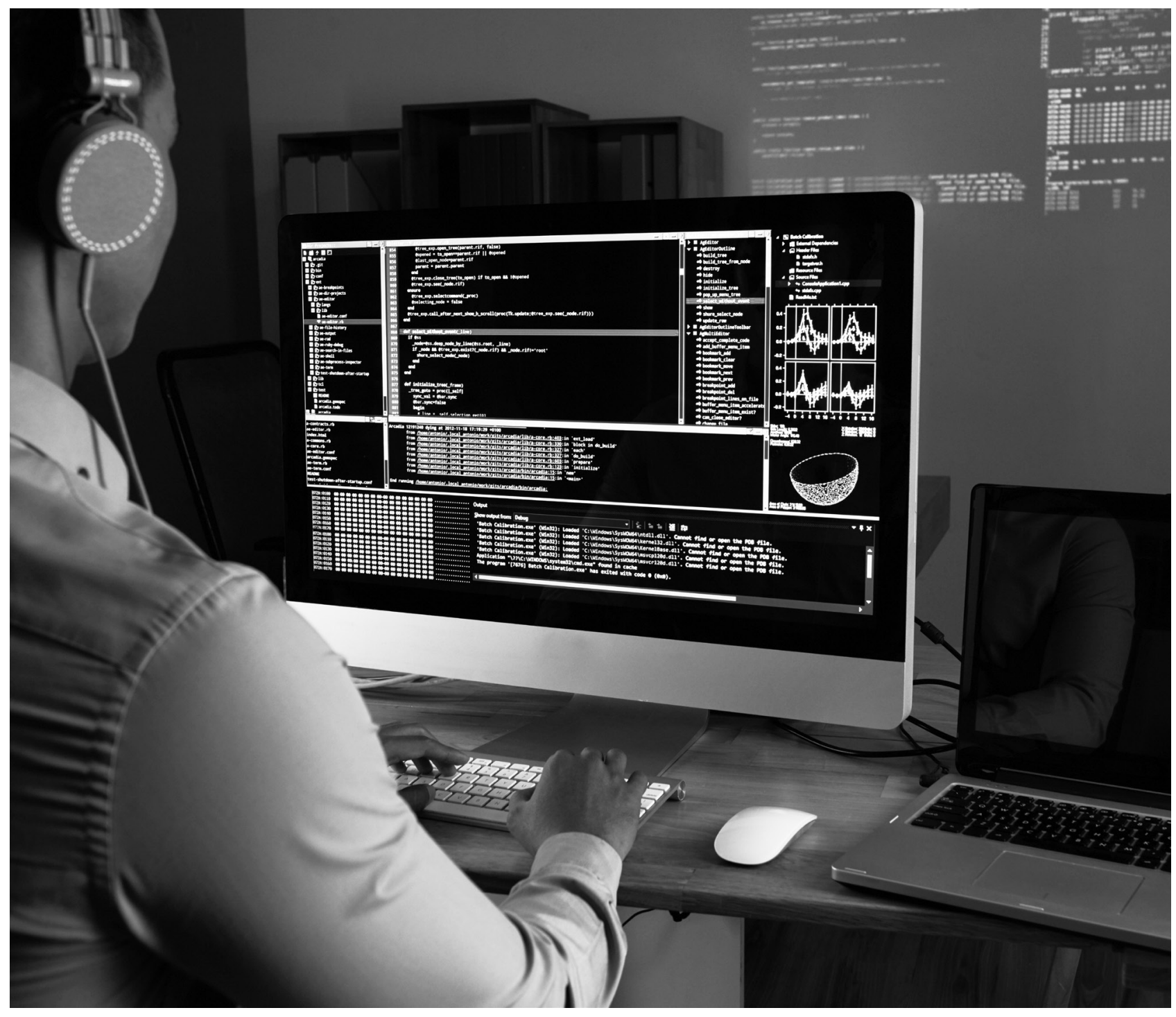




\title{
Desarrollo del pensamiento computacional basado en diseño de tecnología educativa ${ }^{1}$ Development of computational thinking based on the design of educational technology
}

\author{
Rafael Ricardo Mantilla G², Francisca Negre Bennasar ${ }^{3}$
}

Artículo recibido en enero 03 de 2019; artículo aceptado en marzo 04 de 2019.

Este artículo puede compartirse bajo la Licencia Creative Commons Atribución-NoComercial-Compartirlgual 4.0 Internacional

y se referencia usando el siguiente formato: Mantilla, R. \& Negre, F. (2019). Desarrollo del pensamiento computacional basado en diseño de tecnología educativa. I+D Revista de Investigaciones, 14 (2), 75-86. DOI: https://doi.org/10.33304/revinv.v14n2-2019007

\begin{abstract}
Resumen
La era digital y los continuos avances tecnológicos generan cambios en la forma de vivir, actuar y pensar, lo que exige nuevas habilidades para ser competitivo e innovador. Los avances originan nuevas habilidades. Inventos como la rueda, la bombilla, el automóvil, la computadora y el internet fueron estableciendo varios tipos de analfabetismo. Inicialmente el término comprendía la incapacidad de leer y escribir; después, la necesidad de una segunda lengua, y finalmente, destrezas mínimas para atender los retos de la sociedad del conocimiento con nuevos recursos digitales. Para el siglo XXI, se llama alfabetización digital, con cambios obligatorios en la forma de pensar, actuar y resolver problemas. Como respuesta, surge el pensamiento computacional, visto como la capacidad para resolver problemas con apoyo de la tecnología e informática. En el abanico de estrategias para lograr su desarrollo, se presentan aquí los resultados acumulados en dos años, desde un semillero de investigación.
\end{abstract}

Palabras clave: Pensamiento computacional, tecnología educativa, semillero investigación, alfabetización digital.

\begin{abstract}
The digital age and the continuous technological advances generate changes in the way of living, acting and thinking, which demand new skills to be competitive and innovative. The advances originate new abilities. Inventions such as the wheel, the light bulb, the automobile, the computer, and the Internet established various types of illiteracy. Initially, the term included the inability to read and write; then the need for a second language, and finally, minimum skills to meet the challenges of the knowledge society with new digital resources. For the $21 \mathrm{st}$ century, it is called digital literacy, with obligatory changes in the way of thinking, acting and solving problems. The answer is computational thinking, seen as the ability to solve problems with the support of technology and
\end{abstract}

1. Artículo de investigación empírica, con enfoque cualitativo, resultado de un proyecto de investigación culminado, perteneciente al área de Ingenierías, subárea de sistemas, desarrollado en el grupo de investigación GIDSAW y financiado por la Universidad de Investigación y Desarrollo (UDI), de la ciudad de Bucaramanga (Colombia). Dirección: calle 9 n. ${ }^{\circ}$ 23-55, PBX: 5776352525 . Fecha de inicio: febrero de 2017. Fecha de terminación: noviembre de 2018.

2. Magister en Tecnología Educativa, Instituto Tecnológico de Monterrey. Integrante del Grupo de Investigación GIDSAW, Universidad de Investigación y Desarrollo (UDI), Bucaramanga (Colombia): Dirección: calle 9 n. ${ }^{2}$ 23-55, PBX:57 76 352525. ORCID ID: https://orcid.org/0000-00022690-3318. Correo electrónico institucional: rmantilla1@udi.edu.co.

3. Doctora en Ciencias de la Educación. Integrante del grupo de investigación Grupo de Infancia, Tecnología, Educación y Diversidad, Universitat de les Illes Balears, de Palma de Mallorca (España): Dirección: carrera de Valldemossa, km 7,5. Palma (Illes Balears), PBX: +34 - 971173000.0 ORCID ID: https://orcid.org/0000-0003-4636-2675. Correo electrónico institucional: xisca.negre@uib.es. 
computing. In the range of strategies to achieve its development, results accumulated during two years are presented, from a research incubator.

Keywords: Computational thinking, educational technology, research incubator, digital literacy.

\section{Introducción}

Asistimos a una era gobernada por equipos de cómputo que ejercen control y cambian formas de actuar, interactuar y pensar. Las múltiples fuentes de información y la omnipresencia del internet como superautopista de comunicación facilitan y dan ventajas en la distribución, capacidad de almacenamiento, rapidez de publicación y herramientas poderosas de búsqueda de información. Estos medios electrónicos presentan ventajas que van reemplazando el formato de libros, revistas, bibliotecas, entre otros (Galina, 2002).

La historia ha demostrado cómo los inventos marcan las acciones, costumbres, culturas y épocas del hombre, con inventos que van desde la rueda (3500 a. C), la lámpara incandescente (1987), el automóvil (1769), la computadora (1942) y el internet (1969). Esto solo por mencionar unos pocos que han marcado generaciones completas.

Cada época, de igual manera que marca un avance y tendencia en la evolución del hombre y en la forma en que interactúa con el mundo, también trae consigo necesidades de formarse (alfabetización). Y estas necesidades implican dificultades para quienes no están preparados. Para los intereses del artículo llamaremos a estas dificultades "analfabetismo".

\section{Del analfabetismo a la alfabetización}

Delimitamos el concepto de 'analfabetismo' de acuerdo con Braslavsky (2003). Es el opuesto de alfabetismo, que consiste en la habilidad de leer y escribir, con un dominio aceptable para las exigencias de la comunidad.

Basados en lo anterior, y en concordancia con Torres (2006), se puede definir 'analfabetismo' como la incapacidad de leer y escribir. Este concepto se liga generalmente a la falta de aprendizaje. Para Cassany y Castellá (2011), la literacidad o sociocultura de la lectura y escritura no es solo un proceso cognitivo o acto de decodificación, sino que alude a tareas sociales y prácticas culturales en una comunidad hablante.

Entonces, le evolución del hombre y los avances tecnológicos evidencian nuevas incapacidades diferentes a la analfabetización absoluta, que hacía alusión a la incapacidad de leer y escribir.
Posteriormente, ante la globalización y otros conceptos como cibercultura y ciberespacio, que van disminuyendo las fronteras entre los países, nuevos medios de comunicación y tecnologías soportadas en internet surgen como la necesidad de comunicarse con otras culturas. Ello da origen a la aldea global, con nuevas habilidades y tendencias en las personas.

Producto de la globalización, la eliminación de fronteras, los nuevos medios de comunicación, los nuevos modelos de trabajo y otros cambios que generalizan la necesidad de dominar una segunda lengua (generalmente el inglés), surge el analfabetismo laboral. Se relaciona con la necesidad de dominar el inglés como idioma universal, dado que la ausencia de un nivel básico de desempeño trae consigo rechazo social.

Para Navas y Alemán (2009), la alfabetización absoluta (lectora y escritura) y su dominio contribuyen en el dominio de una segunda lengua, por las estructuras y patrones mentales desarrollados.

Actualmente, la sociedad de la información y la participación de los dispositivos de cómputo en las diferentes labores que desarrolla el ser humano han cambiado las formas de interactuar, comunicarnos, trabajar, pensar, sentir y aprender (Guzman \& Gutíerrez, 2018; Simanca, Porras, Garrido \& Hernández, 2017). El desconocimiento de los avances tecnológicos o nuevas tecnologías se conoce como analfabetismo digital (Guaña, Valencia, Topón \& Pérez, 2016). Alude al no saber cómo interactuar digitalmente y obtener beneficios de estos medios, lo que se evidencia en el poco o nulo manejo de herramientas informáticas. Esto afecta a los sujetos en los ámbitos profesional, personal y social.

En el marco de la era digital, de los diferentes sectores de la sociedad y el nivel de la capacidad que interactúan con tecnologías, surge una reciente clasificación de tres grupos: los nativos digitales, que han ido creciendo a la par que las tecnologías; los inmigrantes digitales, que fueron adaptándose a las tecnologías; y los analfabetos digitales, que se mantienen al margen de las tecnologías (Guaña, Valencia, Topón \& Pérez, 2016).

\section{Alfabetización digital}

De acuerdo con Rodríguez y Dolores (2008), la alfabetización digital abre las puertas a la civilización 
digital, lo que permite que existan mejores relaciones de comunicación, laborales, familiares, sociales y culturales.

Czernik (2006) afirma:

"Una verdadera alfabetización digital no consiste solo en enseñar a utilizar una computadora y distintas aplicaciones informáticas, sino que, además, debe ofrecer los elementos básicos para el desarrollo de capacidades que permitan la comprensión y dominio del lenguaje en el que están codificados los programas".

En la búsqueda por alcanzar esta alfabetización, las instituciones de educación pública, privadas, básica, media, secundaria, de pregrado y de posgrado generan diferentes estrategias curriculares y transversales desde la academia, para forjar estudiantes competitivos como futuros ciudadanos y fuerza motriz de un pueblo, cultura, región o país. En su búsqueda por articular estos patrones de enseñanza, llegan a las siguientes preguntas: ¿cómo pensamos?, ¿qué estrategia implementaremos para fomentar uno de estos pensamientos?

\section{Tipos de pensamiento}

Para Vygotsky (1979), en contraste con Bermejo (1998), el pensamiento y el lenguaje están totalmente ligados en aspectos que forman una estructura. Ello desde el significado de la palabra (semántica), estructura del lenguaje (sintaxis), componente pragmático (contexto) y procesos neurológicos (producción).

La combinación de estos elementos (semántica, sintaxis y contexto), la metacognición y su intención de profundización en unos aspectos más que en otros llevan a una determinada clasificación del pensamiento. Para Monereo y Castello (1997), esta clasificación contiene estos aspectos: lógico, crítico, reflexivo, divergente, convergente y creativo.

Se resaltan tres tipos de pensamiento:

- Pensamiento crítico: Entendido como el pensamiento que permite procesar datos y tomar decisiones con base en sus propias creencias, con expresiones de actividad intelectual, orientadas a conseguir objetivos académicos y para la vida diaria. En este tipo de pensamiento se utilizan habilidades relacionadas con el razonamiento, la resolución de problemas y la toma de decisiones (Lara, 2012).

- Pensamiento creativo: Es el responsable de generar nuevas ideas, alternativas e ideas originales. Establece conexiones entre lo que sabe y lo que aprende, y demuestra un aprendizaje significativo, estrechamente relacionado con el pensamiento crítico.

- Pensamiento metacognitivo: Se refiere al grado de conciencia y conocimiento que el individuo tiene de sí mismo sobre la forma de pensar. Consiste en conocer, aprender y resolver problemas.

Marzano (1992) propone ocho actividades, operaciones o destrezas que estimulan el desarrollo del pensamiento crítico como suprapensamiento que afecta los demás de la lista. Estas ocho destrezas son:

- Comparación: para la identificación y articulación de semejanzas y diferencias entre los objetos reales y abstractos.

- Clasificación: para agrupar objetos con base en sus atributos o características.

- Inducción: para inferir generalizaciones, principios, afirmaciones soportadas en procesos de análisis.

- Deducción: para inferir las consecuencias que se pueden desprender al aplicar determinadas generalizaciones 0 principios.

- Análisis de errores: para identificar y articular errores desde el razonamiento.

- Elaborar fundamentos: para diseñar y construir escenarios que permitan comprobar afirmaciones.

- Abstraer: para identificar el patrón o secuencia que subyace dentro de un conjunto de datos.

- Analizar diferentes perspectivas: para valorar y contrastar el propio punto de vista con el de los demás.

En relación con un aprendizaje basado en competencias, Villa y Poblete (2007) incorporan más tipos de pensamientos (ver Figura 1). Para el presente artículo, no es relevante el lóbulo donde se forma, pero sí la representación de los estadios de cómo pensamos.

El pensamiento reflexivo aborda el modo de pensar, ordenar ideas, tomar decisiones conscientes y reconocer la forma de pensar y la mentalidad.

El pensamiento analítico permite catalogar lo que sabemos, a través de comparaciones y de establecer categorías.

El pensamiento sistémico se emplea en ideas con una realidad irreductible, de la que no lo podemos deslindar. Es la aceptación de una realidad previamente modelada y simulada (comprobada).

El pensamiento analógico, con la aplicación de comparaciones mentales no directas, implica el conocimiento de diferentes realidades. Es un esquema mental que compara distintas ideas por el parecido que puedan tener en relación con otras. 
El pensamiento deliberativo es aquel que soporta las decisiones. No hace referencia a cálculo matemático o estadístico, sino al hecho de aplicar el poder de decisión de acuerdo con una serie de valores.

El pensamiento práctico permite discernir y aplicar las mejores soluciones para realizar una acción. Establece qué es lo conveniente, para aplicar mejoras. Por medio del pensamiento práctico, creamos rutinas de trabajo. Es el pensamiento que se usa en la aplicación de una u otra técnica artística, en función del soporte, de la temática o de nuestra habilidad.
El pensamiento grupal se mide desde dos niveles. El nivel del pensamiento adquirido, que se integra en nuestra propia manera de pensar, $y$, por tanto, lo hacemos nuestro; y el pensamiento de un grupo y su utilización por parte de un conjunto de personas (población). Este último es clave en la resolución de posibles conflictos vivenciales.

Por su parte, Castañeda (2000), basado en la lista de habilidades que puede desarrollar el ser humano, aumenta la lista de pensamientos. Añade el pensamiento lógico, creativo, sistémico, estratégico, prospectivo, lateral, difuso, probabilístico, filosófico y ético. Estos se desarrollan en los diferentes lóbulos del cerebro (ver Figura 2).

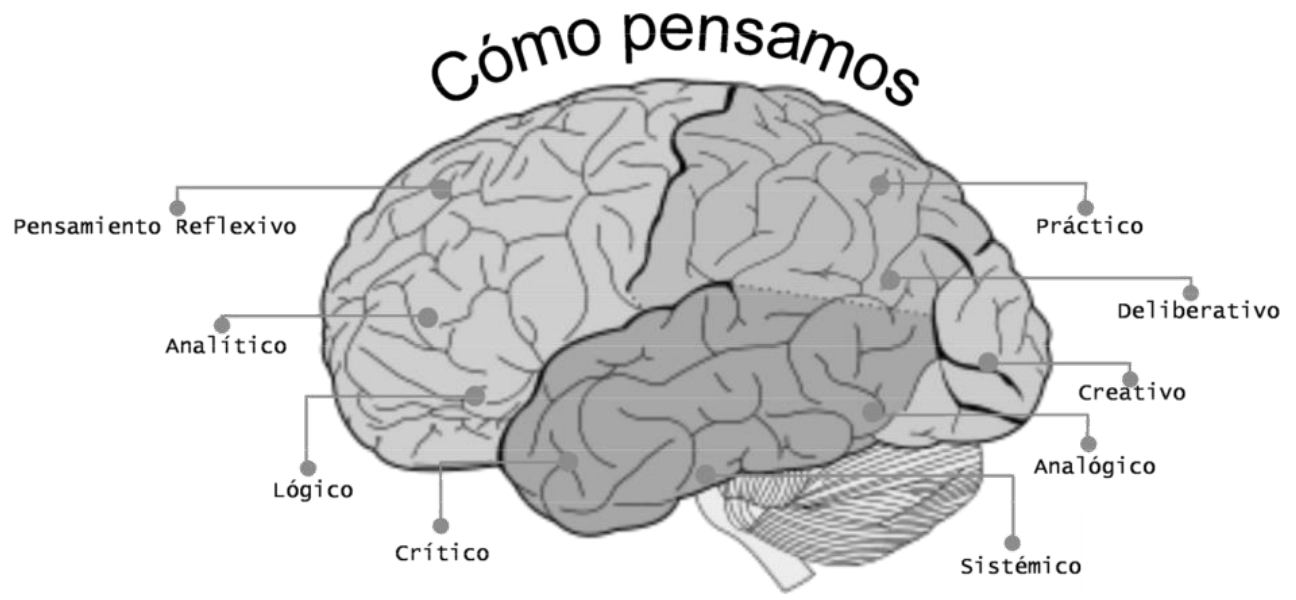

Figura 1. Cómo pensamos. Fuente: Villa y Poblete (2007).

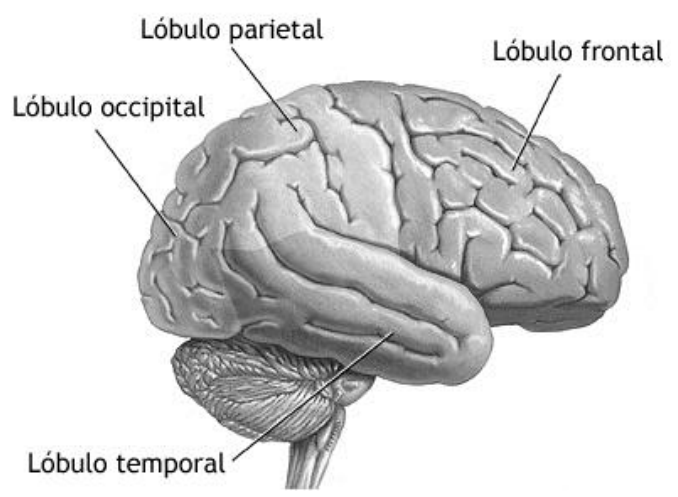

Figura 2. Lóbulos del cerebro. Fuente: MedlinePlus (2018).

En relación con estos tipos de pensamiento, se ciernen demandas específicas, como las de empleadores que representan el contexto laboral. En un estudio adelantado por Villa y Poblete (2007) (ver Tabla 1), se presentan las 10 competencias más valoradas en Reino Unido, Europa y Japón.
Tabla 1

Las 10 competencias más valoradas en el contexto laboral

\begin{tabular}{ccc}
\hline $\begin{array}{c}\text { REINO } \\
\text { UNIDO }\end{array}$ & EUROPA & JAPÓN \\
\hline $\begin{array}{c}\text { Trabajo bajo } \\
\text { presión }\end{array}$ & $\begin{array}{c}\text { Resolución de } \\
\text { problemas: } \\
\text { trabajo } \\
\text { autónomo }\end{array}$ & $\begin{array}{c}\text { Resolución de } \\
\text { problemas }\end{array}$ \\
$\begin{array}{c}\text { Comunicación } \\
\text { oral }\end{array}$ & $\begin{array}{c}\text { Comunicación } \\
\text { oral }\end{array}$ & Ejercicio físico \\
$\begin{array}{c}\text { Precisión y } \\
\text { atención al } \\
\text { detalle }\end{array}$ & $\begin{array}{c}\text { Trabajo bajo } \\
\text { presión }\end{array}$ & $\begin{array}{c}\text { Comunicación } \\
\text { oral: precisión, } \\
\text { atención al } \\
\text { detalle }\end{array}$ \\
Trabajo en & Hacerse & \\
equipo & responsable & \\
& de las & \\
decisiones & Trabajo en & Trabajo en
\end{tabular}




\begin{tabular}{|c|c|c|}
\hline tiempo & equipo & equipo \\
\hline $\begin{array}{l}\text { Capacidad de } \\
\text { adaptación }\end{array}$ & $\begin{array}{l}\text { Asertividad, } \\
\text { persistencia y } \\
\text { decisión }\end{array}$ & $\begin{array}{c}\text { Capacidad de } \\
\text { concentración, } \\
\text { gestión del } \\
\text { tiempo }\end{array}$ \\
\hline Iniciativa & $\begin{array}{c}\text { Capacidad de } \\
\text { adaptación, } \\
\text { iniciativa, } \\
\text { precisión }\end{array}$ & \\
\hline $\begin{array}{c}\text { Trabajo } \\
\text { autónomo }\end{array}$ & & \\
\hline $\begin{array}{l}\text { Hacerse } \\
\text { responsable } \\
\text { de las } \\
\text { decisiones }\end{array}$ & & \\
\hline $\begin{array}{l}\text { Planificación, } \\
\text { coordinación }\end{array}$ & & Iniciativa \\
\hline $\begin{array}{c}y \\
\text { organización }\end{array}$ & & \\
\hline
\end{tabular}

Fuente: Villa y Poblete (2007).

De la Tabla 1, se deriva la importancia de trabajar unas dimensiones más que otras. Por ejemplo, el pensamiento crítico; el pensamiento reflexivo; las habilidades comunicativas; el sentido común, con influencia de la utilización de las TIC; el trabajo desde grupos heterogéneos; las orientaciones del aprendizaje; la comunicación; la aplicación del pensamiento matemático; y la resolución de problemas.

\section{Pensamiento computacional}

Se delimita ahora el concepto de pensamiento computacional (PC). Para Wing (2006), es "el proceso de pensamiento envuelto en formular un problema y sus soluciones, de manera que las soluciones son representadas de una forma en que pueden ser llevadas a un agente de procesamiento de información". Ello como una habilidad fundamental para todos, no solo para los informáticos. Para la lectura, la escritura y la aritmética, deberíamos promover el pensamiento computacional en la capacidad analítica de cada niño.

Unos años después, Valverde-Berrocoso, FernándezSánchez y Garrido-Arroyo (2015) indican que el pensamiento computacional es una competencia básica que todo ciudadano debería conocer, para desenvolverse en la sociedad digital, pero que no es una habilidad "rutinaria"o"mecánica". Es una forma de resolver problemas de manera inteligente e imaginativa, y además posee las características de combinar abstracción y pragmatismo, ya que se fundamenta en las matemáticas.
Como un mecanismo inteligente de aprendizaje para afrontar puntualmente los retos que trae consigo la era digital, así como para apoyar la alfabetización digital, se encuentra el pensamiento computacional. Este pensamiento incorpora habilidades y características del pensamiento crítico, creativo, lógico, analítico, reflexivo, práctico y sistémico, entre otros, desde el plano de las tecnologías educativas (Llorens Largo, García-Peñalvo, Molero Prieto \& Vendrell Vidal, 2017).

El pensamiento computacional se concibe como una metodología que articula conceptos básicos de las ciencias de la computación para resolver problemas cotidianos de forma rápida y efectiva. Se emplea para resolver problemas que no pueden ser tratables de forma sencilla por una persona (Basogain, Olabe \& Olabe, 2015).

Su principal promotora, y quien acuñó el término de pensamiento computacional, fue Wing (2006). Esta autora ha hecho alusión a la nueva forma en que se deben afrontar los problemas basados en el potencial que ofrece la computación. Wing describe con detalle las principales propiedades asociadas al pensamiento computacional (ver Tabla 2).

\section{Tabla 2}

\section{Características del pensamiento computacional}

\begin{tabular}{|c|c|}
\hline N. ${ }^{\circ}$ & Características \\
\hline 1 & $\begin{array}{l}\text { Reformular un problema a uno parecido } \\
\text { que sepamos resolver por reducción; esto } \\
\text { es, encuadrarlo, transformarlo, simularlo. }\end{array}$ \\
\hline 2 & Pensar recursivamente. \\
\hline 3 & Procesar en paralelo. \\
\hline 4 & $\begin{array}{l}\text { Interpretar códigos como datos y datos } \\
\text { como códigos. }\end{array}$ \\
\hline 5 & Generalizar análisis dimensional. \\
\hline 6 & $\begin{array}{l}\text { Reconocer ventajas y desventajas de } \\
\text { solapamiento. }\end{array}$ \\
\hline 7 & $\begin{array}{l}\text { Reconocer coste y potencia de tratamiento } \\
\text { indirecto y llamada a proceso. }\end{array}$ \\
\hline 8 & $\begin{array}{l}\text { Juzgar un programa por simplicidad de } \\
\text { diseño. }\end{array}$ \\
\hline 9 & $\begin{array}{l}\text { Utilizar abstracción y descomposición en un } \\
\text { problema complejo o diseño de sistemas } \\
\text { complejos. }\end{array}$ \\
\hline 10 & $\begin{array}{l}\text { Elegir una correcta representación o modelo } \\
\text { para hacer tratable el problema. }\end{array}$ \\
\hline 11 & $\begin{array}{l}\text { Seguridad en su utilización y en su } \\
\text { modificación en un problema complejo sin } \\
\text { conocer cada detalle. }\end{array}$ \\
\hline
\end{tabular}


12 Modularizar ante múltiples usuarios.

13 Prefetching y caching anticipadamente para el futuro.

14 Prevención, protección, recuperarse de cada escenario en el peor caso.

15 Utilizar razonamiento heurístico para encontrar la solución.

16 Planificar y aprender en presencia de incertidumbre.

17 Buscar, buscar y buscar más.

18 Utilizar muchos datos para acelerar la computación.

19 Límite tiempo/espacio y memoria/potencia de procesado.

Fuente: Basogain, Olabe y Olabe (2015).

Los componentes que articula el pensamiento computacional, según Valverde (2015), se presentan en la Figura 3.

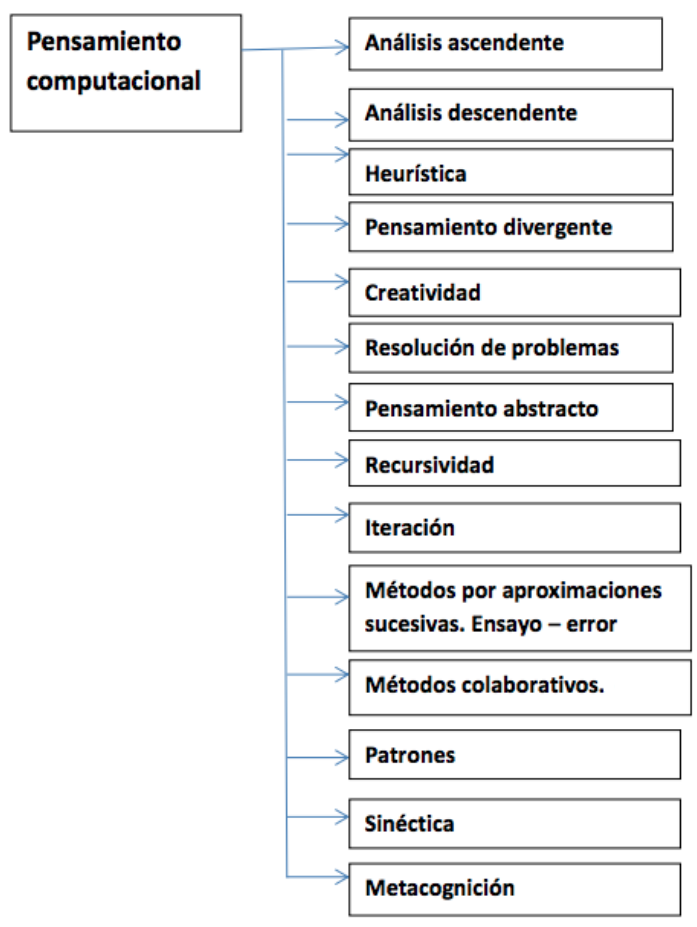

Figura 3. Componentes articulados en el pensamiento computacional. Fuente: Valverde (2015).

Para Basogain, Olabe y Olabe (2015), se deben desarrollar 4 dimensiones como mínimo para alcanzar el desarrollo del pensamiento computacional: descomposición, abstracción, patrones y algoritmos (ver Figura 4).

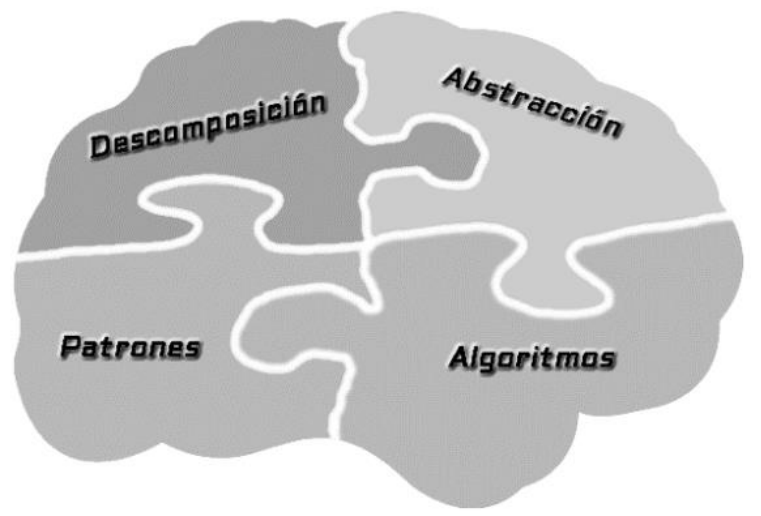

Figura 4: Componentes del pensamiento computacional. Fuente: Autores.

La abstracción hace referencia a identificar las características esenciales del proceso que se desea crear, aislándolo de detalles superfluos.

La descomposición se refiere al proceso por el cual se divide un problema en partes mínimas, para transformar grandes y complejos problemas en pequeños y sencillos problemas por resolver.

Los patrones consisten en buscar similitudes entre diferentes problemas ya resueltos o afrontados anteriormente para ser reutilizados. Cuantos más patrones reconozcan, más fácil y rápida será la tarea de resolver problemas.

Los algoritmos son el plan de acción diseñado para ejecutar instrucciones claras y precisas, en un orden efectivo, para resolver problemas.

Como tarea y compromiso, las instituciones educativas deben buscar estrategias que propicien el desarrollo del pensamiento computacional. Wing (2006) presenta el pensamiento computacional como el conjunto de los procesos de pensamiento implicados en la definición de problemas y en la representación de sus soluciones, de manera que dichas soluciones pueden ser efectivamente ejecutadas por un agente de procesamiento de información (humano, computadora o combinación de ambos).

\section{Metodología}

\section{Tipo de estudio}

La investigación es aplicada y corresponde al tipo experimental. Ello por la manipulación intencional de la variable independiente pensamiento computacional, 
que presenta un nuevo escenario, por la integración de "plataformas de jueces online" y de redes de entrenamiento en programación competitiva. Se busca así una mayor influencia sobre las variables que componen el pensamiento computacional (descomposición, abstracción, patrones y algoritmos). Esto se debe a la propuesta planeada en el modelo pedagógico basado en participación de maratones y torneos de programación competitiva. El enfoque es descriptivo y presenta las características más relevantes con relación al desempeño y los resultados en el ámbito nacional, dentro del calendario de entrenamiento (RPC ${ }^{4}$ y $\mathrm{CCPL}^{5}$ ), así como del encuentro oficial en la maratón nacional de programación, que otorga los cupos para participar oficialmente en la maratón latinoamericana de programación competitiva (ambos organizados por $\mathrm{ACIS}^{6} / \mathrm{REDIS}^{7}$, en representación de la ICPC ${ }^{8}$.

\section{Participantes}

El diseño muestral se da desde un ambiente natural, propio de la sinergia que rodea un semillero de investigación, que corresponde a la participación voluntaria y motivada de cualquier estudiante de la Facultad de Ingenierías de la Universidad de Investigación y Desarrollo. Los estudiantes de esta facultad ven en su malla curricular materias relacionadas con la programación de computadoras (Fundamentos de Programación, Lógica Computacional, Programación I y II, entre otras). También tiene que ver el interés por resolver problemas e incluso mejorar técnicas para su desempeño laboral. El grupo finalmente se registra con 28 estudiantes ( 6 mujeres, 22 hombres), conformado por 8 estudiantes entre $7 .^{\circ}$ y $10 .^{\circ}$ semestre; 6 estudiantes entre $3 .^{\circ}$ y $6 .^{\circ}$ semestre; y 14 estudiantes entre $1 .^{\circ}$ y $2 .^{\circ}$ semestre. Las edades oscilan entre los 17 y los 28 años, con un promedio de 20 años. El escenario de interacción se da con frecuencia semanal (todos los sábados), en horario comprendido entre la 1:00 p. m. y las 6:00 p. m., en un aula con computadoras y asignación por equipo de dos y tres estudiantes (trabajo en equipo). Este último es el requisito que se tiene para participar en los torneos y maratones nacionales por parte de las redes de entrenamiento.

\section{Materiales e instrumentos}

Los recursos empleados en el estudio corresponden a plataformas virtuales de entrenamiento para la gestión, así como para el registro de participantes para los diferentes encuentros establecidos por un cronograma de maratones en dos redes de entrenamiento (RPC y CCPL). Asimismo, corresponden a los jueces online que evalúan el desempeño en el desarrollo de los 12 retos por maratón, para clasificar los equipos con base en sus resultados en los ámbitos nacional (CCPL) e internacional (RPC), y los equipos de cómputo con IDE para programar en lenguaje $\mathrm{C} / \mathrm{C}++\mathrm{y}$ Java.

La recolección de los datos se lleva a cabo desde los jueces online. La publicación de sus resultados se da frente a cada ejercicio o reto que compone la competencia, que llamaremos pruebas estandarizadas (por la rigurosidad de su diseño y estructura).

\section{Pruebas estandarizadas}

Las pruebas que conforman las diferentes maratones tienen una estructura definida: su presentación es en inglés; presentan un título -el nombre que debe llevar el archivo al enviar al juez online-; un enunciado de la situación problema; una breve descripción de las características de los datos de entrada; una descripción sobre los datos de salida; y uno o dos ejemplos para su verificación. Cada reto tiene unos parámetros relacionados con eficiencia y consumo de recursos máximos permitidos para ser aceptado. Estas maratones agrupan en promedio 12 retos, y obedecen a un calendario presentado desde principio de año, con un evento mensual. Es decir, se participó en 2 maratones por mes; sin embargo, a veces el calendario coincidía, y se organizaban dentro del semillero varios equipos participantes ( 3 estudiantes por equipo) para atender a los dos encuentros.

\section{Procedimiento}

Con la intención de alcanzar y generar ambientes para el fomento del pensamiento computacional, existen diferentes estrategias, que van desde la adaptación y reforma al currículo; la atención temprana desde las escuelas; la mediación de los videojuegos; la incorporación de los lenguajes de programación en los planes de aula; el uso de diferentes recursos de la tecnología educativa, como aulas virtuales de aprendizaje; objetos de aprendizaje; microblogs, implementación de LMS, entre otros.

Para el presente artículo, se muestra una estrategia que integra la tecnología educativa, con jueces, ejercicios y entornos de desarrollo integrado de programación online

7. REDIS (Red Colombiana de Programas de Ingeniería de Sistemas y Afines).

8. ICPC (International Collegiate Programming Contest). 
(ver Figura 5), desde un espacio complementario de trabajo (un aula de clase), en horario alterno al académico (los sábados). Se caracteriza por la investigación constante, el trabajo en grupo, la diversidad por la heterogeneidad de sus integrantes, que corresponden a estudiantes de diferentes carreras profesionales y diferentes niveles o semestres de formación.

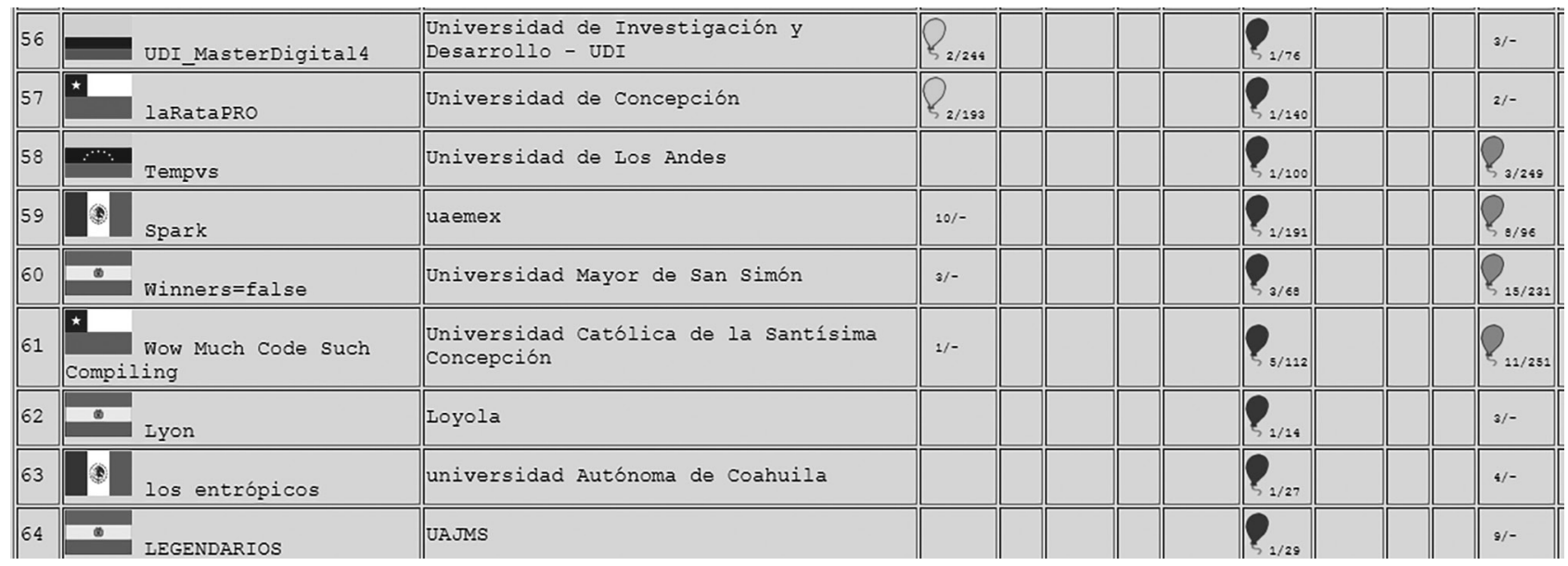

Figura 5. Juez online BOCA. Fuente: Autores.

Todos se reúnen bajo una línea de trabajo llamada "club de programación competitiva", que se basa en la participación constante en maratones y torneos. Obtienen el reconocimiento como sede oficial para la participación del calendario de encuentros; agregan ese ingrediente de competitividad y medición constante, frente a otras universidades reconocidas del contexto nacional e internacional, a través de un semillero de investigación.

El semillero de investigación incorpora dentro de sus recursos de gestión y aprendizaje plataformas de tecnología educativa, para mediar en la resolución de problemas, a través de los dispositivos de cómputo.

Durante dos años de trabajo, se lograron grandes metas, como hacer parte del selecto grupo de universidades avaladas como sedes oficiales para participar en los calendarios de entrenamiento en programación competitiva, por parte de las dos redes más representativas en Colombia: la Red de Programación Competitiva (RPC), perteneciente a la Sociedad Colombiana de Computación (SCo2) (febrero de 2017) y la Colombian Collegiate Programming League (CCPL), perteneciente a la International International Collegiate Programming Contest (abril del 2017) (ver Figuras 6 y 7 ).

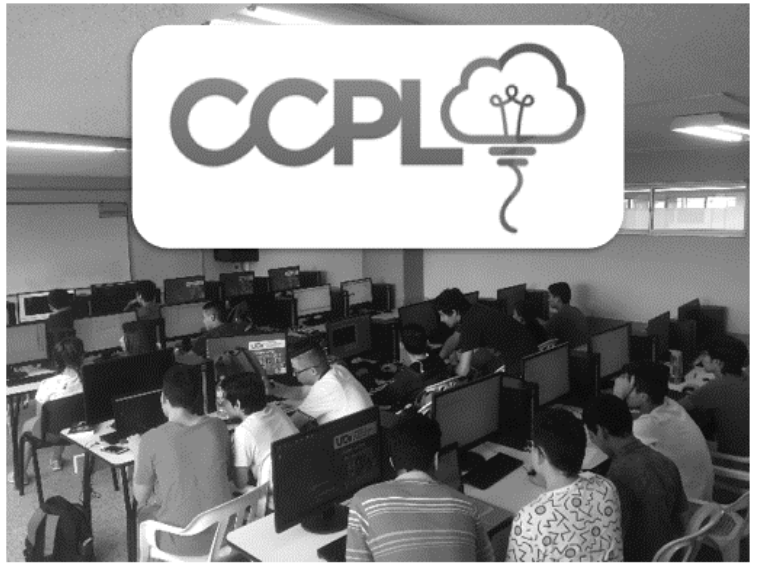

Figura 6. Participación de eventos organizados por CCPL. Fuente: Autores.

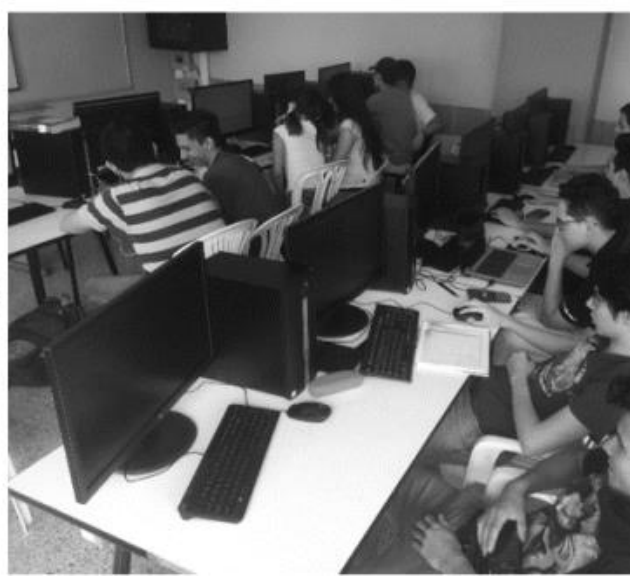

Figura 7. Participación en eventos de la RPC. Fuente: Autores. 


\section{Resultados}

Los resultados se presentan en 3 secciones: RPC, CCPL y ACIS/REDI. Se consideran dos momentos: el primer año de trabajo y el segundo año.

Durante el primer año de trabajo, los resultados en la participación de estas redes de entrenamiento no fue el mejor, porque muchos encuentros no lograban cumplir con un reto, según los parámetros de aceptación por parte del juez online. Además, RPC, a diferencia de CCPL, permitía como entrenamiento, después de la maratón, continuar enviando ejercicios en modo posmaratón. Se establecieron unas metas ambiciosas para el segundo año de estudio.

RPC es una red de entrenamiento que también representa una ventana frente a universidades de diferentes regiones del mundo. Se establecieron como reto para los 10 encuentros 15 ejercicios aceptados dentro del encuentro oficial; se lograron 18, para un cumplimiento de la meta del $120 \%$. Por su parte, en modo posmaratón el reto era de 20 retos adicionales a los desarrollados durante el horario oficial, y se consiguieron 18, para un cumplimiento de la meta del $90 \%$ (ver Figura 8). Lo anterior representa un fuerte insumo de nuevas técnicas de programación, así como un banco de ejercicios para entrenamiento basado en los retos aceptados que se pueden aplicar en los siguientes años.

CCPL es una red de entrenamiento nacional, representante oficial en Colombia de la ICPC, que organiza el mundial de programación competitiva. Esta red, dentro de las características de sus ejercicios, cuenta con una mayor dificultad, referencia y, por ende, los resultados o buen desempeño allí son de mayor dificultad; adicionalmente, en muchas ocasiones los ejercicios son inéditos. En el primer año, los resultados fueron insuficientes, con 3 retos, cifra en que se basa la proyección meta y se establece un plan de trabajo. La meta se fija para participar en los 10 encuentros de 10 retos aceptados, con uno por evento; sin embargo, los equipos consiguen 15 retos, para un indicador de desempeño en cumplimiento de la meta del 150 $\%$. Aunque no se cuenta con modo posmaratón, se buscan jueces online de otros países, para continuar evaluando algoritmos realizados en modo posmaratón. Ello para enriquecer el banco de ejercicios y el área de conocimiento, con una meta de 10 adicionales, de los que se cumplieron 16 nuevos ejercicios, para una meta cumplida del $160 \%$ (ver Figura 9).

ACIS y REDIS organizan el encuentro anual en la XXXII Maratón Nacional de Programación, que da cupo a los mejores 40 equipos en el ámbito nacional para representar a Colombia en la Maratón Regional Latinoamericana ACM-ICPC 2018. Allí, uno de los equipos, con un entrenamiento de 2 años, y con apoyo de tecnología educativa, incrementa sus niveles de desarrollo de pensamiento computacional y supera la prueba con clasificación directa, por su buen desempeño, y pasa a representar a Colombia en un certamen de tan importante reconocimiento (ver Figura 7). De esta forma, se cumple con la meta del $100 \%$ para 3 ejercicios proyectados.

\begin{tabular}{lccc} 
Indicador & Meta & $\frac{\text { Porcentaje Progreso }}{120 \%}$ \\
\hline GLOBOS RPC & 15 & $90 \%$ \\
GLOBOS RPC & 20 & $90 \%$
\end{tabular}

Figura 8. Criterios de evaluación RPC. Fuente: Autores.

\begin{tabular}{lccc} 
Indicador & Meta & $\frac{\text { Porcentaje Progreso }}{120 \%}$ \\
\hline GLOBOS RPC & 15 & $90 \%$ \\
GLOBOS RPC & 20 & 9
\end{tabular}

Figura 9. Criterios de evaluación CCPL. Fuente: Autores. 


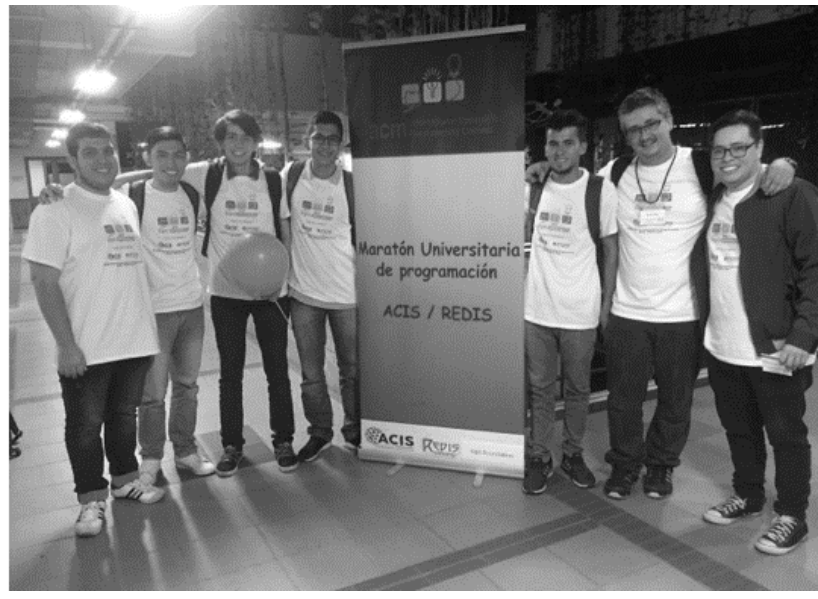

Figura 10. Clasificación de latinoamericanos. Fuente: Autores.

\section{Conclusiones}

Los resultados obtenidos son satisfactorios. Se cuenta con indicadores basados en el desempeño en las maratones y posmaratones que demuestran un buen trabajo. Estos sirven como indicador de que la metodología está funcionando sobre el desempeño de los estudiantes. Estos han pasado del anonimato a ser los únicos representantes de las universidades de Santander y Norte de Santander en obtener cupo directo para representar a Colombia en la competencia latinoamericana, con miras al mundial.

Internamente, si bien el reto o meta que se presentó (2018) parecía ambicioso, al menos con referencia a los resultados del año anterior (2017), estos fueron superados en más del $100 \%$. Ello sirve de fundamento para ampliar los parámetros de proyección para el siguiente año (2019).

El desarrollo del pensamiento computacional se fortalece desde la conformación de un semillero de investigación, bajo la línea de programación competitiva. Si bien no se tiene un número exacto de la muestra, propio de las características que rodean a un semillero que trabaja bajo la filosofía de interés y asistencia voluntaria, se ve un alto compromiso en los participantes en atender los encuentros en un día de descanso (sábado), y aun con una jornada continua y extensa (1 a 6 p. m.).

Se detectan otros beneficios paralelos a la intención principal del estudio, en el desarrollo del pensamiento computacional como competencia indispensable para la era digital. Estos son el fortalecimiento del inglés, la comprensión lectora y el desarrollo del pensamiento matemático.

\section{Agradecimientos}

Se hace mención especial de las redes de entrenamiento CCPL y RPC, por promocionar estos encuentros virtuales, con un cronograma de maratones estricto y sobresaliente organización lógico-técnica. Finalmente, se hace mención de cada uno de los estudiantes que de forma voluntaria creen y hacen parte de este proyecto, quienes han demostrado altos niveles de pertinencia y compromiso.

\section{Referencias}

Basogain, X., Olabe, M., \& Olabe, J. (2015). Pensamiento Computacional a través de la Programación: Paradigma de Aprendizaje. RED. Revista de Educación a Distancia, (46).

Bermejo, V. (1998). Desarrollo Cognitivo. España: Editorial Sínstesis.

Braslavsky, B. (2003). ¿Qué se entiende por alfabetización? Revista Latinoamericana de Lectura. 24(2), 6-23

Cassany, D., \& Castellà, J. (2011). Aproximación a la literacidad crítica. Perspectiva, 28(2), 353-374.

Castañeda, L. (2000). Pensar, tarea esencial de lideres y gerentes / Think, which was Critical of Leaders and Managers: Aplicacion del Pensamiento Multimodal a la Toma de Decisiones y la Solución de Problemas Organizacionales. México: Ediciones Poder.

Czernik, D. (2006). Alfabetos y saberes: la alfabetización digital. Comunicar: Revista científica iberoamericana de comunicación y educación, (26) 78-82.

Galina, I. (2002). La lectura en la era digital. Red de revistas científicas de América Latina, el Caribe, España y Portugal, 5(1), 11-15.

Guaña, E., Valencia, J., Topón, D., \& Pérez, M. (2016). El analfabetismo digital en docentes limita la utilización de los evea. Revista Publicando, 3(8), 24-36.

Guzman, A., \& Gutíerrez, C. (2018). Las competencias digitales y el uso de las tecnologías sociales: el valor agregado en la comercialización en el sector calzado en Bucaramanga. I+ $D$ Revista de Investigaciones, 11(1), 17-27. https://doi.org/10.33304/revinv.v11n12018002

Lara, A. (2012). Desarrollo de habilidades de pensamiento y creatividad como potenciadores de aprendizaje. Revista Unimar, 30(1).

Llorens Largo, F., García-Peñalvo, F. J., Molero Prieto, X., \& Vendrell Vidal, E. (2017). La enseñanza de la informática, la programación y el pensamiento computacional en los estudios preuniversitarios. Education in the Knowledge Society (EKS), 18(2), 7-17.

Marzano, R. (1992). Dimensiones del aprendizaje. México: Instituto Tecnológico de Estudios Superiores de Occidente. 
MedlinePlus. (2018). Lóbulos del cerebro. Retrieved from https://medlineplus.gov/spanish/ency/esp_ imagepages/9549.htm

Monereo, C., \& Castelló, M. (1997). Las estrategias de aprendizaje. España: Edebé.

Nava, G., \& Alemán, R. (2009). Lectura en Lengua Materna (Español) y el Desarrollo de la Producción Oral y Escrita en Inglés. México: Memorias del V Foro de Estudios en Lenguas Internacional.

Rodriguez, M., \& Dolores, M. (2008). Alfabetización digital: el pleno dominio del lápiz y el ratón. España: Universidad de Huelva.

Simanca, F. A., Porras, A. A., Garrido, F. B., \& Hernández, P. C. (2017). Implementación de herramientas tecnológicas en los procesos de enseñanza- aprendizaje de los triángulos. I+D Revista de Investigaciones, 10(2), 79-88. https://doi.org/10.33304/revinv.v10n2-2017006

Torres, R. (2006). Alfabetización y aprendizaje a lo largo de toda la vida. Revista interamericana de Educación de Adultos, 28(1), 25-38.

Valverde, J., Fernández, M., \& Garrido, M. (2015). El pensamiento computacional y las nuevas ecologías del aprendizaje. RED-Revista de educación a distancia, 46(3).

Vygotsky, L. (1979). El desarrollo de los procesos psíquicos superiores. Barcelona: Edit. Crítica.

Villa, A., \& Poblete, M. (2007). Aprendizaje Basado en Competencias. Bilbao: Ediciones Mensajero.

Wing, J. (2006). Computational thinking. Communications of the $A C M, 49(3), 33-35$. 